\title{
Facultés de médecine francophones et responsabilité sociale : approche stratégique 2015-2020
}

\section{French-speaking faculties of medicine and social accountability : 2015-2020 strategic approach}

Paul GRAND'MAISON ${ }^{1}$, Joël LADNER ${ }^{2}$, Ahmed MAHERZI ${ }^{3}$, Geneviève POITEVIEN ${ }^{4}$, Julien POITRAS ${ }^{5}$, Réjean DUPLAIN ${ }^{6}$, Charles BOELEN ${ }^{7}$, José GOMEZ ${ }^{8}$, Tewfik NAWAR ${ }^{1}$, Dominique PESTIAUX ${ }^{9}$, Michel ROLAND ${ }^{10}$, André-Jacques NEUSY ${ }^{11}$

1 Faculté de médecine et des sciences de la santé, Université de Sherbrooke, Canada

2 Faculté de médecine, Université de Rouen, France

3 Faculté de médecine, Université de Tunis El Manar, Tunisie

4 Faculté des sciences de santé, Université de Quisqueya, Port-au-Prince, Haïti

5 Faculté de médecine, Université Laval, Québec, Canada

6 Faculté de médecine, Université de Montréal, Canada

7 Consultant international en système et personnel de santé, Ancien coordonnateur du programme de l'OMS (Genève) des ressources humaines pour la santé

8 Faculté de médecine, Université de Poitiers, France

9 Faculté de médecine et de médecine dentaire, Université Catholique de Louvain, Belgique

${ }^{10}$ Faculté de médecine, Université Libre de Bruxelles, Belgique

11 Training and Health Equity Network (THEnet)

Manuscrit reçu le 17 novembre 2015 ; commentaires éditoriaux formulés aux auteurs le 7 janvier 2016 ; accepté pour publication le 20 janvier 2016

\section{Mots-clés}

Responsabilité sociale ; Facultés de médecine ; Action stratégique
Résumé - Contexte : Les facultés de médecine francophones au niveau mondial (139), conscientes de leurs obligations, s'engagent pleinement envers leur responsabilité sociale avec l'objectif de répondre au mieux aux besoins des populations qu'elles ont le mandat de servir. En 2012, elles ont mis sur pied un projet international de recherche-action sur la responsabilité sociale. Après quelques années de travaux, elles ont récemment élaboré un plan stratégique pour la période 2015-2020 pour confirmer leurs engagements, mieux coordonner les actions, en faire un suivi plus rigoureux et en augmenter l'impact. Exégèse : Le plan se fonde sur trois axes spécifiques d'intervention: l'impact, l'accréditation, les partenariats/collaborations. Ceux-ci sont renforcés par trois axes transversaux de soutien : plaidoyer/formation/expertise, dissémination/leadership, organisation/ressources. Les objectifs de chacun des axes ainsi 
qu'une série d'actions prioritaires pour la période 2015-2017 sont présentés. Conclusion : Ce plan stratégique confirme l'engagement concret des facultés de médecine francophones envers leur responsabilité sociale pour les prochaines années.

Keywords Social accountability; Faculties of medicine; Strategic action
Abstract - Context: Worldwide, French-speaking faculties of medicine (139) are conscious of their obligations and fully committed with respect to social accountability in order to optimally respond to the needs of populations they are mandated to serve. In 2012, they launched an international action research project on social accountability. After a few years of work, they recently put together a strategic plan for 2015-2020 to confirm their commitments, coordinate their action in an optimal way, improve the thoroughness of action follow-up and improve its impact. Analysis: The plan is based on three specific intervention axes: impact, accreditation, partnerships/collaborations. They are supported in three transversal support areas: advocacy/training/expertise, dissemination/leadership, organization/resources. The objectives of each axis as well as a series of priority actions for 2015-2017 are presented. Conclusion: The strategic plan reaffirms the commitment of the French-speaking faculties of medicine regarding social accountability in the coming years.
«Au sujet de l'avenir, il ne s'agit pas de le prévoir mais de le rendre possible. » Antoine de Saint-Exupéry

\section{Contexte}

Dans le contexte actuel de mondialisation et des besoins de santé multiples non comblés, la nécessité de transformer l'éducation, la recherche et la pratique médicale ne fait plus de doute ${ }^{[1]}$. Les facultés de médecine sont particulièrement interpellées à cet égard, elles dont les obligations sociales et éthiques dérivent d'une part des buts pour lesquels la société les a établies et les soutient, notamment au regard du degré avec lequel elles servent vraiment ces buts ${ }^{[2]}$. C'est dans ce contexte qu'est de plus en plus mis en avant un plaidoyer international pour des facultés plus responsables, plus conscientes des populations à servir et de leurs besoins, plus engagées pour que leurs actions, notamment celles reliées à la formation des futurs professionnels de la santé, aient un impact véritable sur les services de santé et la santé des individus et des populations ${ }^{[3-4]}$.

Le mouvement international actuel portant sur la responsabilité sociale des facultés de médecine exige donc une redéfinition de la mission des facultés de médecine et une réorientation de leurs actions. Elle exige aussi une mesure de l'impact de leur engagement envers la responsabilité sociale sur leur fonctionnement et sur leur contribution à la réponse aux besoins des populations à servir et à l'amélioration de la santé de la population.

Dans ce contexte, et tel que recommandé par le Consensus mondial sur la responsabilité sociale des facultés de médecine ${ }^{[5]}$, il y a nécessité de plaider de façon continue en faveur de la responsabilité sociale des facultés de médecine, d'expérimenter son adaptation et son application dans différents contextes, d'effectuer des activités de recherche à cet égard, de développer des normes permettant de mesurer et illustrer la responsabilité sociale, de coordonner aux niveaux national et international des projets de responsabilité sociale et d'en assurer la diffusion.

Ces dernières années, plusieurs initiatives ont été mises en place au niveau international pour promouvoir la responsabilité sociale : le Training for Health Equity Network -THEnet ${ }^{[6]}$, le projet ASPIRE de l'Association for Medical Education in Europe (AMEE) sur la responsabilité sociale ${ }^{[7]}$, la révision des normes d'évaluation pour y intégrer la responsabilité sociale par la World Federation for Medical Education (WFME) ${ }^{[8]}$, l'engagement de l'Association des facultés de médecine du Canada (AFMC) en regard de la responsabilité sociale ${ }^{[9]}$. 


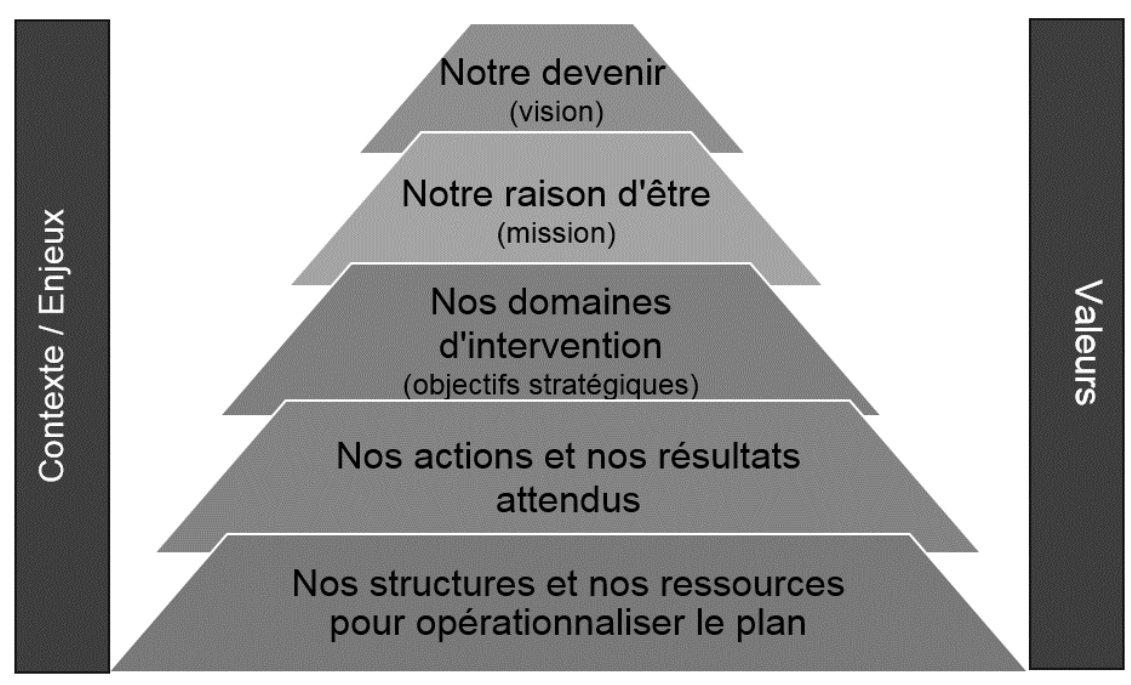

Fig. 1. Éléments de planification stratégique.

C'est dans ce cadre que ce situe le projet international de recherche-action des facultés de médecine francophones sur la responsabilité sociale, mis en place par la Conférence internationale des doyens et des facultés de médecine d'expression française (CIDMEF) et la Société internationale francophone d'éducation médicale (SIFEM). Ce projet, initié au début de l'année 2012, implique maintenant 56 facultés francophones réparties dans 18 pays à travers le monde.

\section{Vers un plan stratégique 2015-2020}

En 2014-2015, après plus de deux ans d'actions multiples, les facultés de médecine francophones engagées dans ce projet ont entrepris une démarche de planification stratégique pour la période 20152020. Le processus a permis de confirmer plusieurs éléments du projet (figure 1). Il visait l'élaboration d'une stratégie documentée, plus ambitieuse et plus structurée, permettant de mieux confirmer les orientations et objectifs du projet, d'identifier et coordonner ses actions, d'en faire un suivi plus rigoureux et d'en augmenter l'impact. L'élaboration du plan stratégique, coordonnée par le secrétariat exécutif du projet, a inclus de nombreuses consultations auprès des facultés participantes, des organisations responsables du projet, des partenaires de celui-ci et d'autres parties prenantes. Le plan stratégique a été confirmé en juin 2015. Il représente une feuille de route précise pour la période 2015-2020, qui sera en appui au développement et à la gestion du projet, et qui en assurera un impact plus grand aux niveaux facultaire, local, national et international, tout comme sa pérennité à long terme.

\section{Valeurs, vision, mission}

De multiples valeurs liées à la responsabilité sociale ont été identifiées; le projet actuel a fait siennes plusieurs de celles-ci :

- vision globale de la santé et de ses déterminants individuels et populationnels ;

- équilibre entre les valeurs de qualité, équité, pertinence et efficience en santé ;

- engagement envers les populations en besoin, vulnérables et mal-desservies ;

- reconnaissance des soins de santé primaires comme base du système de santé et de la complémentarité entre les soins de première, deuxième et troisième ligne ;

- nouveaux rôles des professionnels de la santé, notamment la collaboration interprofessionnelle ;

- facultés de médecine comme acteurs clés du système de santé ; 


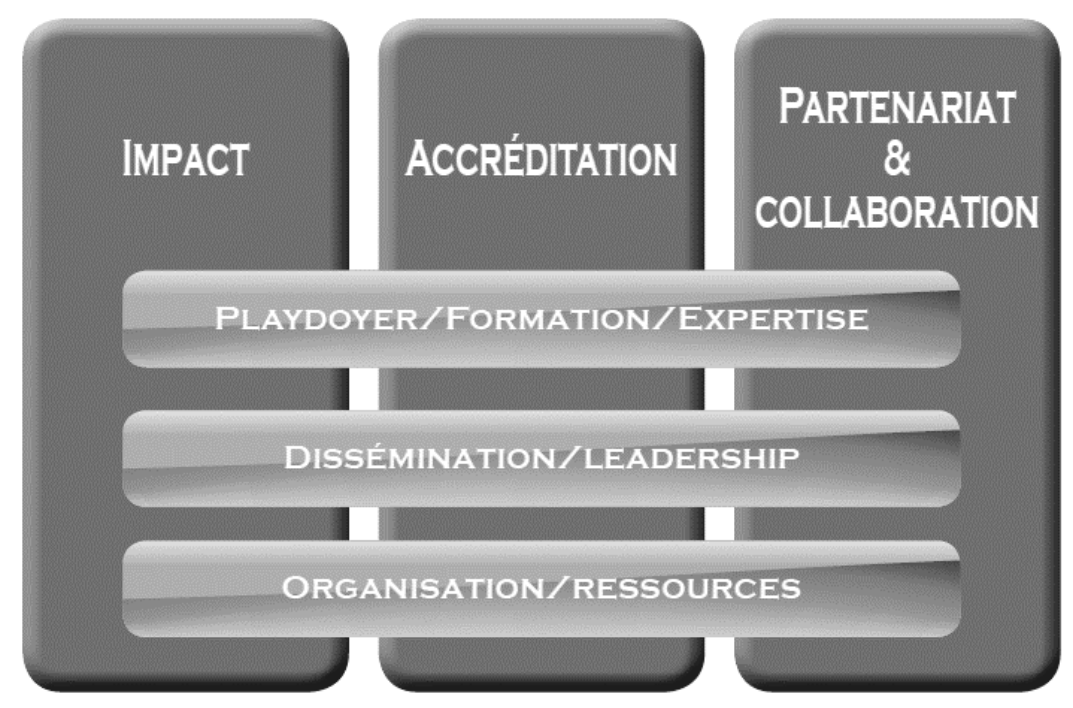

Fig. 2. Plan stratégique $2015-2020$ du projet international francophone de responsabilité sociale des facultés de médecine : axes d'intervention.

- partenariats ;

- évaluation et mesure d'impact des actions ;

- accréditation/certification, un levier puissant d'amélioration continue ;

- vision globale, action locale.

La vision du projet est celle d'être « un promoteur et un agent de changement transformationnel auprès des facultés de médecine et leurs membres, pour qu'elles adoptent la responsabilité sociale comme principe directeur de leur mission et leurs actions, au bénéfice de la santé des communautés et populations pour lesquelles elles s'engagent. »

Sa mission est celle de «mobiliser les connaissances et les ressources dans le but d'appuyer les facultés dans leur engagement envers la responsabilité sociale ».

\section{Orientations stratégiques et actions}

Tel que présenté sur la figure 2, les actions prioritaires du plan stratégique 2015-2020 du projet international francophone sur la responsabilité sociale des facultés de médecine sont organisées selon trois axes spécifiques d'intervention : l'impact, l'accréditation, les partenariats et les collaborations. Ces actions sont renforcées par trois axes transversaux de soutien: plaidoyer/formation/expertise, dissémination/leadership, organisation/ressources.

\section{Impact}

L'engagement d'une faculté de médecine envers sa responsabilité sociale a un impact significatif sur son fonctionnement, sa mission, ses valeurs, ses actions. Cet impact doit être mesuré. De façon toute aussi importante, on reconnait la nécessité de mesurer, en tenant compte des principes de la responsabilité sociale, l'impact des actions des facultés sur les professionnels de la santé qu'elles forment et leur pratique professionnelle, sur le système de santé, sur la réponse aux besoins de santé des individus et des populations desservies tout comme sur leur état de santé ${ }^{[10-11]}$.

Le projet a fait siens les objectifs suivants :

- promouvoir et soutenir l'utilisation d'un cadre de référence et de mesure de la responsabilité sociale;

- partager avec d'autres organisations des outils de mesure d'impact ;

- développer des expertises de mesure d'impact ; 
- confirmer les impacts à mesurer pour la formation médicale.

\section{Accréditation}

L'évaluation et encore plus l'accréditation représentent un levier majeur pour orienter la mission et les actions d'une faculté. Le projet fera donc la promotion de l'accréditation des facultés de médecine et, plus spécifiquement, de la prise en compte de la responsabilité sociale dans les normes et critères d'accréditation $^{[12-13]}$.

Les objectifs du projet pour la période 2015-2020

à l'égard de l'accréditation sont :

- effectuer un plaidoyer pour l'accréditation des facultés ;

- soutenir les facultés/pays pour la mise en place de mécanismes et agences d'accréditation ;

- élaborer des normes et critères de responsabilité sociale pour l'accréditation ;

- œuvrer à intégrer la responsabilité sociale dans les normes et critères d'accréditation.

\section{Partenariats et collaborations}

Intégrer la responsabilité sociale dans les facultés de médecine pour mieux répondre aux besoins de santé des populations ne peut se faire seul. Les partenariats et les collaborations sont des conditions essentielles et souvent gages de succès. À cet égard, le présent projet maximisera les collaborations liées à la responsabilité sociale avec des organisations nationales et internationales. Il fera la promotion de partenariats entre les facultés de médecine, et entre cellesci et les principaux intervenants du système de santé, notamment les responsables politiques, les gestionnaires de santé, les professionnels de la santé, quelle que soit leur discipline, les autres institutions académiques et les populations elles-mêmes ${ }^{[14]}$.

Le projet s'engage donc pour la période 20152020 à :

- développer une collaboration avec d'autres organisations en regard de la responsabilité sociale ;

- encourager les facultés à travailler ensemble sur des projets de responsabilité sociale ;

- promouvoir un cadre de référence pour les partenariats.

\section{Plaidoyer/formation/expertise}

Au-delà du plaidoyer pour la responsabilité sociale, la formation des intervenants facultaires et le développement d'expertise dans ce domaine sont des priorités que reconnaissent les responsables du présent projet. De fait, elles sont à la base du succès pour l'atteinte des objectifs des axes d'intervention précités.

Le projet a donc comme objectif de :

- recueillir, produire et diffuser des documents de plaidoyer et d'information sur la responsabilité sociale ;

- répertorier et partager les meilleures pratiques de responsabilité sociale ;

- développer et offrir des activités de formation de niveau initial et avancé portant sur la responsabilité sociale ;

- soutenir la participation d'étudiants, professeurs et gestionnaires facultaires.

\section{Dissémination/leadership}

Sans la diffusion des expériences mises en place et des expertises développées, le projet n'aura pas l'impact voulu tant auprès des facultés participantes et des organismes regroupant des facultés de médecine qu'auprès de la communauté scientifique internationale. Le projet vise donc à maximiser la diffusion de ses actions et expertises, à développer des projets de recherche en responsabilité sociale et à se positionner au niveau national et international comme leader en responsabilité sociale.

Les objectifs spécifiques 2015-2020 sont les suivants :

- assurer la diffusion scientifique des expertises du projet et des facultés participantes ;

- implanter des activités de recherche sur la responsabilité sociale et la mesure de l'impact des facultés ;

- assurer la reconnaissance internationale du projet comme un leader dans le domaine de la responsabilité sociale.

\section{Organisation/ressources}

La mise en place du présent plan stratégique ne pourra vraiment être réussie sans une optimisation de 
l'organisation du projet, et une gestion efficace et efficiente des ressources.

Le projet se fixe donc les objectifs suivants :

- mettre en place des modalités de suivi annuel du plan stratégique 2015-2020 ;

- maximiser la contribution des membres des facultés dans la structure du projet ;

- doter le projet de ressources humaines administratives et de soutien;

- déposer des demandes de financement auprès d'organisations nationales et internationales ;

- soutenir les facultés dans leur recherche de financement.

\section{Actions prioritaires}

Dans chacun des axes d'intervention précités, le plan stratégique 2015-2020 identifie une série d'actions prioritaires pour la période $2015-2017^{[15]}$. Les membres du secrétariat exécutif ont identifié les actions prioritaires pour lesquelles ils s'engagent à œuvrer dans les deux prochaines années (20152017) :

- utiliser des outils d'évaluation d'impact des facultés et de leurs actions et en tirer des leçons ;

- œuvrer à intégrer la RS dans les normes d'évaluation ;

- soutenir les partenariats entre les facultés d'un même pays ;

- développer des actions concrètes de partenariat ;

- mettre en place une plateforme web de partage d'information ;

- offrir des activités de formation selon les besoins des groupes ;

- publier 3 ou 4 articles sur le projet dans son ensemble ou sur des projets facultaires de responsabilité sociale ;

- établir un groupe de recherche, identifier des thèmes prioritaires et concrétiser un projet ;

- consolider la gouvernance du projet ;

- obtenir les ressources humaines et financières nécessaires ;

- mettre en place une conférence internationale sur la responsabilité sociale en 2017 (Tunisie).

\section{Discussion}

L'élaboration d'un plan stratégique est un exercice souvent périlleux mais toujours nécessaire. Comment vérifier que l'ensemble des éléments est considéré ? Comment s'assurer que les contenus du plan sont les plus pertinents ? Comment s'assurer d'un engagement de l'ensemble des parties prenantes à contribuer à ce plan ?

Le processus suivi pour l'élaboration du présent plan présente plusieurs points forts : une consultation large, une expérience de quelques années du projet, des efforts multiples de clarification des éléments du plan, une approche systématique, une implication des parties prenantes, une diffusion large des résultats. Néanmoins, des points faibles existent : des possibilités de compréhension sous-optimale pour les personnes n'ayant pas participé à l'élaboration du plan, une liste d'objectifs pouvant sembler trop exhaustive, la présence de certains recoupements dans les axes d'intervention et objectifs.

Malgré les limites possibles, l'utilisation d'un plan stratégique permet déjà et permettra de mieux orienter les actions du projet. Depuis la confirmation du plan au printemps 2015, toutes les activités mises en place par le projet tiennent compte de celui-ci et de ses axes d'intervention.

Dans le but d'assurer la pertinence continue du plan stratégique, celui-ci sera revu en 2018 pour assurer sa réappropriation par l'ensemble des intervenants, une réorganisation si nécessaire et surtout une nouvelle confirmation des actions prioritaires à accomplir.

\section{Conclusion}

En 2015, la responsabilité sociale est une obligation morale que les facultés de médecine ne peuvent plus mettre de côté ; il en va de leur crédibilité et de leur légitimité vis-à-vis de la société. Dans une position privilégiée pour orienter le développement des connaissances tout comme des ressources humaines, pour influencer positivement l'évolution du système 
de santé et contribuer à l'amélioration de l'état de santé de la population, elles ne peuvent se dérober à cette obligation. La responsabilité sociale donne un sens à l'engagement des facultés au service des populations et individus qu'elles ont le mandat de servir.

La responsabilité sociale n'est pas « un tout ou rien » qui serait atteint à un certain moment ou à un autre. Il s'agit plutôt d'un processus transformationnel continu. La mise en place du plan stratégique du projet international francophone sur la responsabilité sociale des facultés de médecine permettra de contribuer au niveau des facultés, des pays et internationalement à ce que chacune des facultés puisse « être et devenir toujours plus socialement responsable ».

\section{Contributions}

Tous les auteurs sont membres du secrétariat exécutif du projet international francophone de responsabilité sociale des facultés de médecine. Ils ont tous participé activement à la mise en place du projet depuis 2012 et à l'élaboration du plan stratégique 2015-2020 présenté ici.

\section{Remerciements}

Nous tenons à remercier toutes les personnes qui ont participé à Paris, le 2 et 3 février 2015, à une réunion de travail confirmant l'élaboration de ce plan stratégique.

Les travaux liés à l'élaboration du plan stratégique ont été soutenus par une subvention provenant de la Conférence internationale des doyens et facultés de médecine d'expression française (CIDMEF) et de la Société internationale francophone d'éducation médicale (SIFEM).

La version préliminaire de ce plan stratégique a été présentée en avril 2015 à Bruxelles dans le cadre du Vème congrès international francophone de pédagogie des sciences de la santé, organisé par la Société internationale francophone d'éducation médicale (SIFEM).

\section{Déclaration d'intérêts}

Aucun auteur ne déclare de conflit d'intérêts en lien avec le contenu de cet article

\section{Approbation éthique}

Non sollicitée car sans objet

\section{Références}

1. Frenk J, Chen L, Bhutta ZA, Cohen J, Crisp N, Evans $\mathrm{T}$, et al. Health professionals for a new century; transforming education to strengthen health systems in an interdependent world. Lancet 2010;376:1923-58.

2. Pellegrino Edmund D. Humanism and the physicians. Knoxville: The University of Tennessee Press, 1979.

3. Boelen C, Heck J. Définir et mesurer la responsabilité sociale des facultés de médecine, Genève : OMS 2000 [Online]. Disponible sur : http://whqlibdoc.who.int/hq/1995/WHO_HRH_95.7_fre.pdf.

4. Contribution collective de la conférence pour le consensus mondial sur la responsabilité sociale des facultés de medecine. Consensus mondial sur la responsabilité sociale des facultés de médecine. Pédagogie Médicale 2011;12:37-48

5. Gibbs T, McLean M. Creating equal opportunities: the social accountability of medical education. Med Teach 2011;33:620-5

6. Training for Health Equity Network (THEnet).The Social Accountability Framework for Health Workforce Training 2015 [On-line] Disponible sur : http://thenetcommunity.org/social-accountability-framework-background/

7. .ASPIRE. International Recognition of Excellence in Education 2016 [On-line] Disponible sur : http:// www.aspire-to-excellence.org

8. World Federation of Medical Education. Guidelines for the use of WFME Standards. 2007 [On-line] Disponible sur : http://www.wfme.org 
9. Association des Facultés de Médecine du Canada. Responsabilité sociale et enseignement de la médecine au Canada: Le devoir d'un médecin canadien envers la société. 2012 [On-line] Disponible sur: https://www.afmc.ca/fr/outils$\% \mathrm{C3} \%$ A9lectroniques $/ \mathrm{m} \% \mathrm{C3} \%$ A9decins-canadiens-de-demain/introduction

10. Training for Health Equity Network. Cadre d'évaluation de THEnet pour un enseignement professionnel de la santé socialement responsable, Version 1, Monographie (1ère édition). 2011 [On-line] Disponible sur : http://www.thenetcommunity.org

11. Leinster S. Evaluation and assessment of social accountability in medical schools. Med Teach 2011;33:673-6

12. Boelen C, Grand'Maison P, Ladner J, Pestiaux D. Responsabilité sociale et accréditation. Une nouvelle frontière pour l'institution de formation. Pédagogie médicale 2008;9:235-44.

13. Boelen C, Dharamsi S, Gibbs T. The social accountability of medical schools and its indicators. Educ Health (Abingdon) 2012;25:180-94

14. Boelen C. Vers l'unité pour la santé. Défis et opportunités des partenariats pour le développement de la santé. Genève: OMS 2002 [Online]. Disponible sur: http://whqlibdoc.who.int/hq/ 2001/WHO_EIP_OSD_2000.9_fre.pdf

15. Projet international francophone sur la responsabilité sociale des facultés de médecine : http://www.responsabilite-sociale-francophone.org

Correspondance et offprints: Paul Grand'Maison, 3001, $12^{\mathrm{e}}$ avenue Nord, Sherbrooke (Québec), J1H 5N4, Canada Mailto : Paul.Grandmaison@USherbrooke.ca 